\title{
An intraoperative motor tract positioning method in brain tumor surgery: technical note
}

\author{
Fumio Yamaguchi, MD, PhD, ${ }^{1,2}$ Hirotomo Ten, MD, PhD, ${ }^{3}$ Tadashi Higuchi, MD, ${ }^{2}$ \\ Tomoko Omura, MD, ${ }^{4}$ Toyoyuki Kojima, MD, PhD, ${ }^{5}$ Koji Adachi, MD, PhD, ${ }^{6}$ \\ Takayuki Kitamura, MD, PhD, ${ }^{6}$ Shiro Kobayashi, MD, PhD, ${ }^{4}$ Hiroshi Takahashi, MD, PhD, ${ }^{7}$ \\ Akira Teramoto, MD, PhD, ${ }^{2,8}$ and Akio Morita, MD, $\mathrm{PhD}^{2}$
}

\begin{abstract}
1'Department of Neurosurgery for Community Health and 'Department of Neurological Surgery, Nippon Medical School, Tokyo; ${ }^{3}$ Department of Judo Therapy, Faculty of Health Care, Teikyo Heisei University, Tokyo; ${ }^{N}$ Neurological Institute, Nippon Medical School Chiba Hokusoh Hospital, Inzai; ${ }^{5}$ Department of Neurosurgery, Yotsuya Medical Cube, Tokyo; ${ }^{6}$ Department of Neurological Surgery, Nippon Medical School Musashi-Kosugi Hospital, Kawasaki; ${ }^{7}$ Department of Neurosurgery, Kasugai Rehabilitation Hospital, Fuefuki; and ${ }^{8}$ Tokyo Rosai Hospital, Tokyo, Japan
\end{abstract}

\begin{abstract}
Intraoperative 3D recognition of the motor tract is indispensable to avoiding neural fiber injury in brain tumor surgery. However, precise localization of the tracts is sometimes difficult with conventional mapping methods. Thus, the authors developed a novel brain mapping method that enables the 3D recognition of the motor tract for intrinsic brain tumor surgeries. This technique was performed in 40 consecutive patients with gliomas adjacent to motor tracts that have a risk of intraoperative pyramidal tract damage. Motor tracts were electrically stimulated and identified by a handheld brainmapping probe, the NY Tract Finder (NYTF). Sixteen-gauge plastic tubes were mounted onto the NYTF and inserted in the estimated direction of the motor tract with reference to navigational information. Only the NYTF was removed, leaving the plastic tubes in their places, immediately after muscle motor evoked potentials were recorded at the minimum stimulation current. Motor tracts were electrically identified in all cases. Three-dimensional information on the position of motor tracts was given by plastic tubes that were neurophysiologically placed. Tips of tubes showed the resection limit during tumor removal. Safe tumor resection with an arbitrary safety margin can be performed by adjusting the length of the plastic tubes. The motor tract positioning method enabled the 3D recognition of the motor tract by surgeons and provided for safe resection of tumors. Tumor resections were performed safely before damaging motor tracts, without any postoperative neurological deterioration.
\end{abstract}

https://thejns.org/doi/abs/10.3171/2017.5.JNS162978

KEY WORDS brain mapping; brain shift; DTI; motor tract; NY Tract Finder; tractography; oncology; surgical technique

I DENTIFICATION of neural tracts is indispensable for the protection of functions during brain tumor surgery. Among intrinsic brain tumors, gliomas are often not well-demarcated and are hard to distinguish from normal structures. Early identification of neural tracts gives good spatial orientation for surgeons and contributes to the avoidance of neural tract injury. Fiber tracking with diffusion tensor imaging (DTI) has been used to draw the pyramidal tract. However, the lines drawn in tractography do not necessarily show the actual pyramidal tract in terms of the fiber bundle size. ${ }^{7}$ When the tumor is adjacent to the pyramidal tract, tracking of the pyramidal tract is difficult because of the influence of peritumoral edema. Also, information obtained by tractography is often used in conjunction with anatomical data for neuronavigation, but it is difficult to obtain accurate anatomical location of the tract with a navigation system due to brain shifts after craniotomy.

Thus, a real-time neurophysiological technique is necessary for the identification of neural tracts because DTI information in a neuronavigator is not reliable. Intraoperative brain mapping is widely used in the surgery of gliomas

ABBREVIATIONS BIS = bispectral index; DTI = diffusion tensor imaging; GTR = gross-total resection; mMEP = muscle motor evoked potential; NYTF = NY Tract Finder; STR = subtotal resection.

SUBMITTED December 5, 2016. ACCEPTED May 30, 2017.

INCLUDE WHEN CITING Published online November 24, 2017; DOI: 10.3171/2017.5.JNS162978. 
in eloquent brain. The conventional neurophysiological examination is sequential stimulation on the wall of tumor resection. Keles et al. ${ }^{6}$ reported that patients whose subcortical pathways were identified with stimulation mapping were more prone to develop an additional motor deficit than those in whom subcortical pathways could not be identified (27.5\% vs $13.1 \%$ ). Carrabba et al. ${ }^{2}$ also reported the same tendency of new permanent postoperative motor deficits in each group (6.5\% vs 3.5\%). Thus, identification of the subcortical motor tract has been increasing the risk of permanent deficits using the conventional subcortical mapping technique. Smith et al. ${ }^{13}$ and Ulmer et al. ${ }^{15}$ reported reduced diffusion with immediate postoperative MRI around tumor resection cavities that were believed to be ischemic changes. In some cases new postoperative deficits correlated well with the anatomical region of ischemia. Therefore, new postoperative motor deficits may be partly caused by ischemic change due to injury to tiny perforating arteries and/or medullary veins. To avoid the ischemic change of motor tracts, it is important to establish sufficient safety margins. However, with the conventional mapping method, resection is either within or very close to the motor tracts $(2-3 \mathrm{~mm}$ according to previous reports) when they are detected. The setting of an arbitrary safety margin is the best method to prevent ischemic change of the motor tract.

Previous reports found some relationship between the electrical intensity and the distance to the neural tract from the stimulation point: ${ }^{5,11}$ however, the actual distance may vary in each case, probably because of the heterogeneous impedance of normal brain tissue, edematous brain tissue, and tumor tissue..$^{10,20}$ Inaccurate measurement of this distance may result in excessive resection of the adjacent tissue containing important neural tracts or insufficient resection of tumor due to fear of tract injury.

To address this problem, we developed the needle electrode "NY Tract Finder (NYTF)" (Fig. 1), ${ }^{17,18}$ which can be used to identify neural fibers in advance of the resection of tissue near important neural pathways. The advantageous function of this electrode is the measurement of the distance between the stimulated point and the insertion site. Because this electrode skips the intervening tissue that is electrically heterogeneous between the stimulation point and the neural tract, imprecise estimation of distance is avoidable. However, we did experience a problem that once the electrode was removed, the location of the stimulated point was ambiguous and the distance between the stimulated point and the insertion site was changing during tumor resection. To solve this problem, we developed the motor tract positioning method. With this technique, the stimulated point can be marked with plastic tubes that alert surgeons to important neural tracts.
In this paper we introduce this technique and describe safe tumor resection adjacent to important neural pathways, as well as analyze its role in intrinsic brain tumor surgeries.

\section{Method \\ Study Patients}

This technique was performed in 40 consecutive patients with newly diagnosed intrinsic brain tumors adjacent to motor tracts that have a risk of intraoperative pyramidal tract damage. All patients were unwilling to accept any postoperative deterioration in neurological symptoms. Therefore, written informed consent was obtained in all patients according to hospital ethics guidelines after the explanation about the risk of residual tumor intentionally left to avoid worsening of motor function. No ethics committee approval was required for this study because it was a retrospective work concerning an intraoperative technique routinely used in our institution.

\section{Tumor Size}

The approximate size of tumor was measured on MRI or $\mathrm{CT}$ as ellipsoid volume using the following formula:

$$
\text { Tumor volume }\left(\mathrm{mm}^{3}\right)=\pi / 6(\mathrm{~L})(\mathrm{W})(\mathrm{H})
$$

in which $\mathrm{L}=$ length $(\mathrm{mm}), \mathrm{W}=$ width $(\mathrm{mm})$, and $\mathrm{H}=$ height $(\mathrm{mm})$.

Enhanced mass was measured in cases of high-grade glioma, and T2-weighted FLAIR mass was measured in low-grade glioma.

\section{Extent of Resection}

Extent of resection was classified into 3 categories: gross-total resection (GTR, $>90 \%$ tumor removal), subtotal resection (STR, resection of 50\%-90\% of tumor), and partial resection $(<50 \%$ tumor removal).

\section{Diffusion Tensor Imaging}

Motor tracts in each case were visualized by Medtronic Stealth Viz. The 3D relationship and the distance between tumor and the pyramidal tract were evaluated prior to surgery. The DTI information was integrated into the neuronavigation system (Medtronic StealthStation) and used to guide electrode insertion.

\section{Anesthesiology}

Total intravenous anesthesia was performed with propofol and remifentanyl. The bispectral index (BIS) was monitored so that the dose of anesthetics could be adjusted

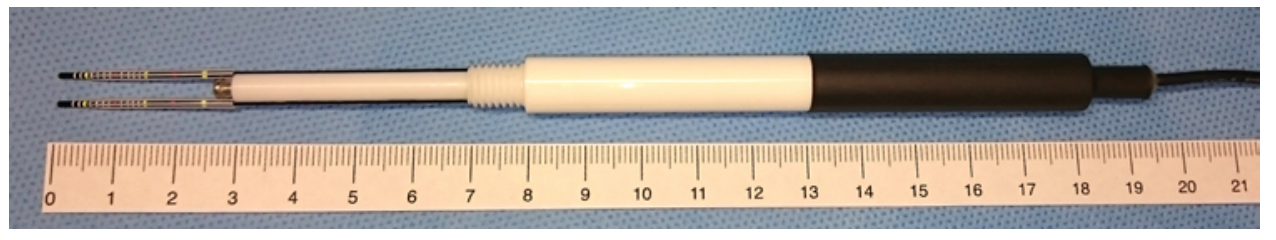

FIG. 1. Photograph of the NYTF with 16-gauge plastic tubes mounted on 2 electrode needles. 
to achieve appropriate depth of anesthesia. A BIS index of between 40 and 60 proved optimal for stable muscle motor evoked potential (mMEP) monitoring. ${ }^{16}$

\section{Tumor Resection}

All glioma resections were performed with navigational guidance using contrast-enhanced T1-weighted MRI along with T2-weighted MRI as appropriate. When the resection reached the point approximately $20 \mathrm{~mm}$ from the motor tract according to DTI data in the navigation system, subcortical motor mapping was started in each case as described below. The resection technique was based on sulcus opening and gyrectomy, with identification and dissection of the sulcus adjacent to the tumor followed by en bloc tumor resection according to the depth at the end of the sulci.

\section{NY Tract Finder}

The bipolar needle electrode of the NYTF has two $30-\mathrm{mm}$-long stainless needles, $1.0 \mathrm{~mm}$ in diameter (Fig. 2). The 2 needles are $5 \mathrm{~mm}$ apart. Each needle is insulated with epoxy except for the distal $1 \mathrm{~mm}$. Each millimeter of the needle's length is marked with a narrow white line, every $5 \mathrm{~mm}$ with a yellow line, and each centimeter with a broad red line (Fig. 2). The electrodes and the handle are connected with a rigid joint, ${ }^{18}$ modified from the flexible copper wire of an original NYTF. ${ }^{17}$ This rigid body enables the operator to achieve stable straight insertion into brain tissue. This electrode NYTF was named after the initials of the Nippon Medical School and an inventor (the name Yamaguchi [Fumio Yamaguchi]). ${ }^{17}$ It was registered with a Japanese patent (registration no. 4686772; the right to a patent was assigned and transferred by the inventor to Nippon Medical School; therefore, the inventor has no financial benefit from the sale of this electrode by the manufacturer). The current NYTF is a modified type of NYTF for navigation-assisted brain mapping, with a rigid joint and a needle diameter larger than the original NYTF (Unique Medical Co., Ltd). This electrode is manufactured for research use and used according to the regulations at each hospital. Currently more than 20 hospitals in Japan, China, and Taiwan are using this electrode.

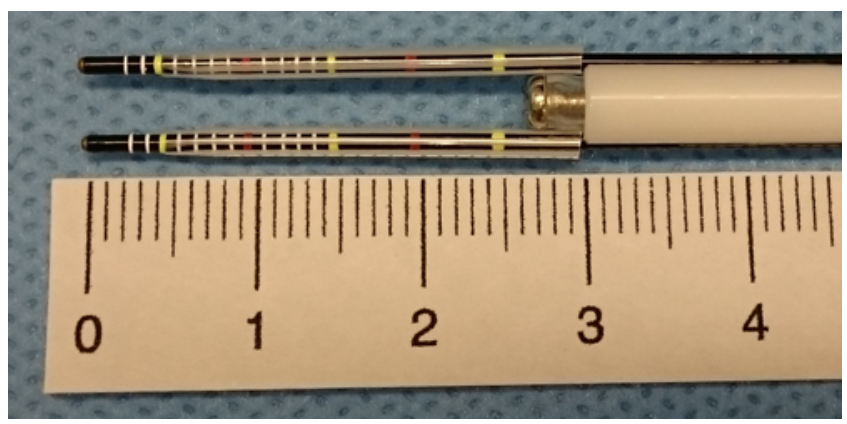

FIG. 2. Electrode needles of the NYTF. The 2 needles are $5 \mathrm{~mm}$ apart. Each needle is insulated with epoxy except for the distal $1 \mathrm{~mm}$. Each millimeter of the needle's length is marked with a narrow white line, every $5 \mathrm{~mm}$ with a yellow line, and each centimeter with a broad red line. The plastic tube was cut to a length of $25 \mathrm{~mm}, 5 \mathrm{~mm}$ short of the electrode length.

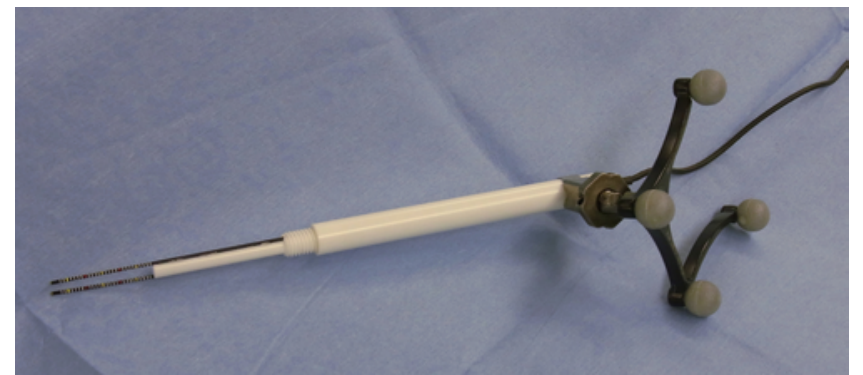

FIG. 3. The NYTF was registered for the navigation system. The midpoint of electrode tips was registered as a tip of the navigation probe.

To register the NYTF with the neuronavigation system, reflective passive marker spheres of the navigation system were attached to a handle of the NYTF and the midpoint of the electrode tips was registered as the tip of probe (Fig. 3).

\section{Navigation-Assisted Subcortical Electrical Stimulation}

Tumor resection was suspended when it approached within approximately $20 \mathrm{~mm}$ from the motor tract, followed by navigation-assisted subcortical electrical stimulation. The details of this procedure have been previously reported ${ }^{17}$ and are briefly described below.

\section{Electrical Stimulation and mMEP Recording}

Electrical stimulation was performed with a neurophysiological monitoring apparatus (Neuropack, Nihon Kohden). The electrical stimulation was $50-$ or $60-\mathrm{Hz}$ pulses of biphasic square-wave waveform of 1-msec pulse width. For cortical electrical stimulation, current intensity is routinely set at approximately $1 \mathrm{~mA}$ to begin and gradually increased by increments of 0.5 to $10 \mathrm{~mA}$; the current is applied for a few seconds for cortical stimulation. If a positive response is achieved, the area is marked with a numbered or lettered tag. The current that activated the mMEP is used as the beginning current for subcortical electrical stimulation.

The same monitoring system was also used as an electromyogram recorder. mMEP was recorded through 8 channels with 16 needle electrodes inserted into the following muscles: face, orbicularis oris; shoulder, trapezius upper and deltoid middle; upper arm, triceps lateral head and biceps brachii; forearm, extensor capri radialis and flexor capri radialis; hand, abductor pollicis brevis and abductor digiti minimi; upper leg, vastus lateralis and biceps femoris short head; lower leg, tibialis anterior and gastrocnemius lateral head; foot, flexor hallucis brevis and flexor digiti minimi. ${ }^{17}$

\section{Subcortical Electrical Stimulation}

Tumor resection is performed following identification of the primary motor cortex and confirmation of the anatomical relationship between the motor cortex and the tumor. When alerted by the neuronavigator of an approximately $20-\mathrm{mm}$ distance to the motor tract, subcortical mappings were performed.

The NYTF is inserted into the wall of the tumor re- 
section cavity in the estimated direction of the tract with image guidance from the neuronavigator. The parameters of electrical stimulation are the same as those for cortical stimulation. Starting with the minimum current that activated mMEPs by cortical stimulation, the electrode is gradually inserted. Then the insertion is stopped when the mMEP of any site of the body is elicited. As a next step, the current intensity is reduced by $0.5-0.1 \mathrm{~mA}$ increments. Then the electrode is inserted again and stopped when an mMEP is elicited. This procedure is repeated until the tip of the electrode is believed to reach the closest position to the motor tract with minimum stimulating current that elicits mMEPs. The depth of the point can be read by the scale on the electrode.

\section{Procedures of the Motor Tract Positioning Technique}

Plastic sheaths for the 16-gauge needle were cut $25-\mathrm{mm}$ long, $5 \mathrm{~mm}$ shorter than the electrodes, setting $5 \mathrm{~mm}$ as the safety margin. This length of plastic tube is arbitrarily set as needed to create a safety margin. Then, cut plastic sheaths were mounted onto each needle of the electrode. Only the NYTF is withdrawn, leaving the plastic tubes in place when the electrode is thought to have reached the closest point to the motor tract (Fig. 4A). When the electrode was withdrawn, the plastic tubes were held by forceps at the beginning. Because the inner diameter of the plastic tube is slightly larger than the outer diameter of the electrode, there is no resistance between them. The placed plastic tubes did not easily move deeper because the blunt tip of each plastic tube has a certain resistance to brain parenchyma. There were some rare cases in which the plastic tubes came out; in these cases, electrode insertion was repeated to put the tubes in the right place.

The bipolar needle electrode was routinely used for subcortical mapping, and fence-post tube placement is routinely used for marking the tumor margin. Therefore, placement of plastic tubes itself is not a new technique. We introduced the method of how to place the plastic tube for the determination of the tumor resection limit. This procedure is performed at several points on the cavity wall on the side of the motor tract. Tumor tissue is resected until the tips of plastic tubes appear (Fig. 4B).

\section{Neurological Assessment}

The assessments of neurological functions were per- formed by 3 neurosurgeons preoperatively and postoperatively (F.Y., H. Ten, and T.H.)

\section{Results}

\section{Patient Characteristics}

Forty patients ( 29 men and 11 women) with lesions in motor-eloquent locations underwent resection during this study. The median age of patients at the time of surgery was 55.8 years (range $24-79$ years). There were 37 highgrade gliomas (9 WHO Grade III and 28 WHO Grade IV) and 3 low-grade gliomas (WHO Grade II). Median tumor size was 55,281.6 $\mathrm{mm}^{3}$ (range 2239.3-2,266,545.0 $\mathrm{mm}^{3}$ ). Symptoms at presentation were hemiparesis in 16 , seizures in 6 (5 generalized and 1 focal), headaches in 6 , sensory disturbances in 2, and memory disturbance in 6 patients. Another 4 patients were asymptomatic and incidentally found.

\section{Detection of Motor Tracts}

mMEPs were elicited and recorded in all cases by electrical stimulation of subcortical motor tracts. In all cases the minimum intensity of electrical current that elicited mMEPs of the motor tract was $0.1-1 \mathrm{~mA}$. White matter stimulation was started at $5 \mathrm{~mA}$ in most cases. During insertion of the electrode, every time mMEPs were detected, stimulation intensity was reduced by $0.5 \mathrm{~mA}$. An electrode was inserted further to obtain the higher mMEP amplitude. mMEPs could be observed until the stimulation currents were reduced to as low as 0.5 or $0.1 \mathrm{~mA}$.

\section{Motor Tract Positioning Technique}

Cut plastic tubes, mounted onto each needle of the electrode, were left in place by withdrawing the NYTF when the electrode was believed to reach the closest point to the motor tract at the minimum electrical current. This procedure was performed at several points on the cavity wall on the side of the motor tract (Fig. 5). The number of plastic tubes ranged from 2 to 8 according to the area of the cavity wall. Surgeons could recognize the 3D localization of the motor tract under the brain surface and the tumor resection cavity (Fig. 4A). Safe tumor resection was performed until the tips of plastic tubes appeared, mainly using an ultrasound aspirator (Fig. 4B).
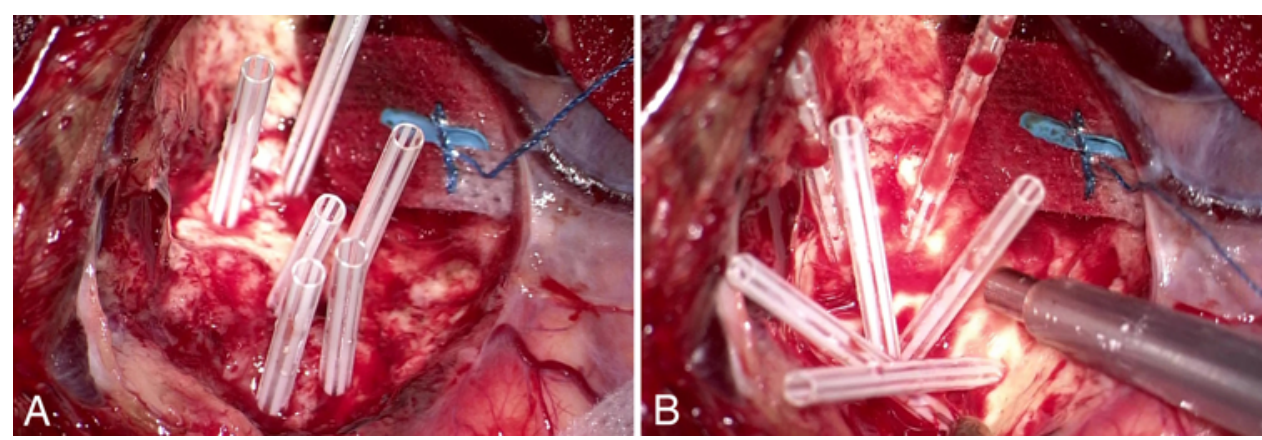

FIG. 4. Tumor resection phase of the motor tract positioning method. A: Plastic tubes were left on the cavity wall. Surgeons could recognize the 3D localization of the motor tract under the brain surface and the tumor resection cavity. B: Safe tumor resection was performed until the tip of the plastic tubes appeared, mainly using an ultrasound aspirator. 

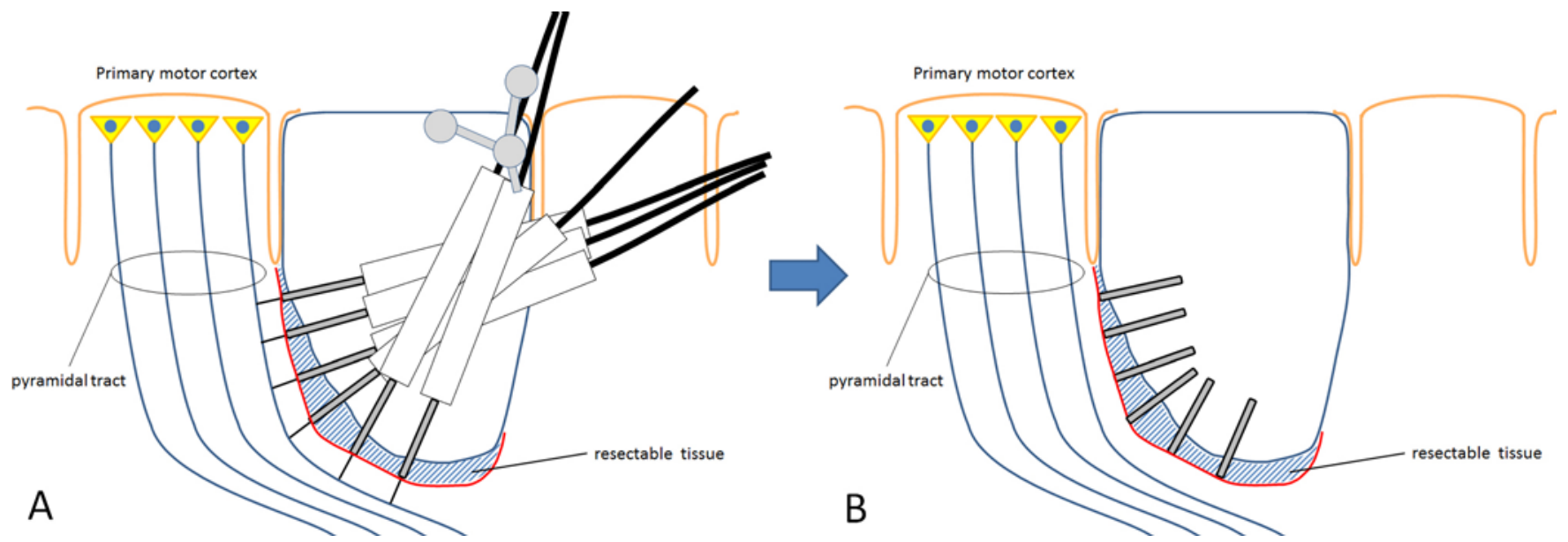

FIG. 5. Illustration of the motor tract positioning method. Cut plastic sheaths, mounted onto each needle of the electrode, were left in place by withdrawing the NYTF when the electrode was believed to reach the closest point to the motor tract at the minimum electrical current (A and B). This procedure was performed at several points on the cavity wall on the side of the motor tract. Copyright Fumio Yamaguchi. Published with permission.

\section{Completeness of Resection}

Tumor resection was performed according to microsurgical standards. GTR was possible and achieved in $82.5 \%$ of cases (33/40), STR in $10 \%$ of cases (4/40), and PR in $7.5 \%$ of cases (3/40). Postoperative mean tumor size was $6939.8 \mathrm{~mm}^{3}$ (range $0-284,533.3 \mathrm{~mm}^{3}$ ).

\section{Postoperative Neurological Function}

There were 3 patients who presented with transient motor weakness and recovered to their preoperative level within 1 month. The cause of this weakness appeared to be Todd's palsy due to intraoperative seizure, supplementary motor area syndrome, or perifocal edema. At 1 month postoperatively, no patient $(0 / 40,0 \%)$ showed permanent deterioration of motor function.

\section{Discussion}

Intraaxial tumors such as malignant gliomas are often not well demarcated and are difficult to distinguish from normal structures. Neuronavigation systems and DTI technologies enable us to recognize the position of certain neural tracts, but the brain deforms once a craniotomy has been performed and the absolute positional information is no longer accurate. To preserve neural functions, surgeons cannot rely on this obscure information. In contrast, neurophysiological measures such as brain mapping and subcortical electrical stimulation can inform us regarding the existence of functioning structures when mMEPs are elicited.

\section{Estimation of the Distance to Neural Tracts}

Efforts to estimate the distance between the stimulation intensity $(\mathrm{mA})$ and the distance $(\mathrm{mm})$ to the motor tract have been reported..$^{11,12}$ There has been a report of a certain tendency for linear correlation between these distances. The relationship is not precise, however, and is not reliable for use in determining the resection margin in each patient. Especially when tumor resection is approaching the cor- ticospinal tract, the correlation of distance is not linear, ${ }^{12}$ so frequent subcortical stimulations are necessary. The reasons why the estimated distance varies in every case include a nonlinear relationship; ${ }^{12}$ heterogeneity of tissue in terms of electrical impedance; ${ }^{3}$ or the changes in electrical response of axons depending on physiological conditions, such as blood flow, blood pressure, blood rheology, carbon dioxide, body temperature, and regional temperature. ${ }^{1} \mathrm{We}$ often experience the increase of stimulation threshold by hypothermia, which increases blood propofol concentration during a constant rate of infusion. ${ }^{8}$ Thus, the conventional method should be used carefully for the estimation of the distance according to surface stimulation during a close approach to neural tracts.

\section{Needle Electrode Insertion}

In contrast, our method for detecting motor tracts is performed by on-site electrical stimulation at the proximity of the neural tract by inserting the needle electrode. The insertion of the electrode, which is often performed in functional neurosurgical operations, is not a standard method in brain tumor surgery.

\section{Bipolar Electrode}

Direct cortical stimulation with bipolar electrodes was found to be very effective in producing localized current flows. Unipolar cortical stimulation, which has a more distant reference electrode, produced higher current densities at the same stimulating current as did bipolar stimulation, but stimulated a larger region of the cortex. ${ }^{9}$ The use of a bipolar electrode in this procedure is based on a previous report. ${ }^{4}$ Studies using optical imaging of bipolar cortical stimulation in the monkey corte ${ }^{4}$ have shown precise local changes (i.e., within 2-3 mm). A graded increase in the area of activation was observed as the stimulation intensity was gradually increased. Therefore, further insertion of the electrode is necessary to shorten the distance between electrodes and axonal fibers to elicit the mMEPs. By repeating this procedure, the position of the electrode 
tip reaches the closest point to axonal fibers. The electrode insertion was stopped when mMEPs were elicited at a certain current intensity. Because of the close proximity of these paired electrodes, the extent of the current field produced by them is limited and more accurate anatomical localization of the site of stimulation becomes possible. Considering electrical field spread by higher current intensity, a small stimulation area is possible by reducing current intensity.

\section{Electrically Heterogeneous Tissues}

Malignant tissues differ from normal tissues (such as brain, breast, skin, prostate, liver, bladder, etc.) in electrical properties, generally attributed to the different water content in neoplastic tissue due to the variation of protein hydration and vascularization, as well as to the membrane permeability, amount of extracellular fluid, packing density, and orientation of the malignant cells. ${ }^{20}$ Sometimes the current field may be distorted by low resistance pathways and this channeling of the current may cause stimulation at unwanted sites. This factor may affect variability of measured distances. Therefore, such demerits are minimized by using bipolar stimulation in which both electrodes are positioned close to each other and have approximately the same conducting contact area. ${ }^{3}$ Also, the aim when inserting the electrode close to the pyramidal tract is the avoidance of electrically heterogeneous tissues. By insertion of the electrode, the tips of the electrode can reach the neural tracts, skipping the intervening electrically heterogeneous tissues.

\section{Waveform Type}

The type of waveform of the electrical stimulation affects the distance from the axon to the electrode. Historically, a rectangular biphasic wave has been used for electrical stimulation for brain mapping and we have been using it following the previous protocol. ${ }^{19}$ This conventional rectangular biphasic pulse activates only nearby axons, while double-exponential waveforms can recruit targeted fibers and change the location of a target by manipulating stimulus intensity. ${ }^{14}$ Therefore, a rectangular biphasic wave is adequate to minimize the area of stimulation.

\section{Inserting Multiple Tubes Into the Vicinity of Motor Tracts}

The problem with our electrode insertion method had been a temporality of neural tract recognition. Despite the fact that accurate identification of tracts was accomplished, the positions of the recognized neural tracts could not be marked once the electrode was removed. The reading of the scale on the electrode gave distance information to surgeons; however, it was changing because tissue removal and frequent stimulations were necessary to know the most current distance. To resolve this inconvenience, stimulated points needed to be marked. The tip of the electrode could be marked with the same length of plastic tube to show the actual site of a neural tract. We usually make plastic tubes that are $5 \mathrm{~mm}$ shorter than the electrodes to keep 5-mm-thick tissue adjacent to the motor tract as a safety margin in our method. This safety margin thickness could be arbitrarily set as needed. By observing multiple plastic tubes left in situ, the direction and the depth of the motor tract could be recognized by surgeons. Tumor resection can be safely performed until the tip of tube appears, because they show the points $5-\mathrm{mm}$ prior to the motor tract. This 3D recognition of the motor tract during the surgical approach enables safe resection without injuring this important functional structure.

\section{Advantages and Disadvantages of This Technique}

Based on the fact that the actual distance between the stimulation point and the neural tract varies in every case, ${ }^{5,10,11,20}$ there may be an inaccurate measurement of its distance using the conventional electrical stimulation method on the wall of the resection cavity. The conduction of electrical stimulation from the wall of the resection cavity has to pass the heterogeneous tissues, e.g., tumor, edematous white matter, nonedematous white matter, and edematous and nonedematous gray matter. Considering the difference of impedance of these tissues, transmission of electrical energy through them is not necessarily homogenous and the electric field intensity in the vicinity of the electrodes becomes nonuniform. Hence, electrical power that is necessary to evoke a certain neural axon is different depending on the intervening tissues between electrode and axon. In contrast, in our method, the tips of the electrode can make their closest approach to the axon. This close placement of the electrode can minimize the thickness of these intervening electrically heterogeneous tissues and consequently it may minimize the electrical power and the variation of stimulating currents necessary for evoking mMEPs.

There might be a possibility of tissue damage and vascular injury by the insertion of the needle electrode. However, no hemorrhage has been experienced by our method so far, and presumably the round shape of the electrode tip can avoid piercing vascular structures.

Awake surgery is also a beneficial technique to detect any rapid neurological change on site. However, even with awake surgery, the condition for the detection of motor tracts is not different regarding the sudden neurological change by vascular injuries. Without electrical detection of the tracts prior to the resection of tumor, unintended excessive resection could occur, resulting in the injury of motor tracts. And even if the structure of the tracts remained, their ischemic change by vascular damage makes motor function deteriorate. This change could occur suddenly; therefore, gradual resection while observing patients' neurological change could fail to protect against neurological deteriorations.

\section{Conclusions}

The motor tract positioning method enables surgeons to recognize the $3 \mathrm{D}$ position of motor tracts and provide safer tumor resection. Further investigation is necessary to elucidate the precision of this method in terms of real proximity of electrical stimulation.

\section{References}

1. Bithal PK: Anaesthetic considerations for evoked potentials monitoring. J Neuroanaesth Crit Care 1:2-12, 2014 
2. Carrabba G, Fava E, Giussani C, Acerbi F, Portaluri F, Songa $\mathrm{V}$, et al: Cortical and subcortical motor mapping in rolandic and perirolandic glioma surgery: impact on postoperative morbidity and extent of resection. J Neurosurg Sci 51:45-51, 2007

3. Delgado JMR: Electrodes for extracellular recording and stimulation, in Nastuk WL (ed): Electrophysiological Methods, Part A. Physical Techniques in Biological Research, Volume V. New York: Academic Press, 1973, pp 89-143

4. Haglund MM, Ojemann GA, Blasdel GG: Optical imaging of bipolar cortical stimulation. J Neurosurg 78:785-793, 1993

5. Kamada K, Todo T, Ota T, Ino K, Masutani Y, Aoki S, et al: The motor-evoked potential threshold evaluated by tractography and electrical stimulation. J Neurosurg 111:785-795, 2009

6. Keles GE, Lundin DA, Lamborn KR, Chang EF, Ojemann G, Berger MS: Intraoperative subcortical stimulation mapping for hemispherical perirolandic gliomas located within or adjacent to the descending motor pathways: evaluation of morbidity and assessment of functional outcome in 294 patients. J Neurosurg 100:369-375, 2004

7. Kinoshita M, Yamada K, Hashimoto N, Kato A, Izumoto S, Baba T, et al: Fiber-tracking does not accurately estimate size of fiber bundle in pathological condition: initial neurosurgical experience using neuronavigation and subcortical white matter stimulation. Neuroimage 25:424-429, 2005

8. Leslie K, Sessler DI, Bjorksten AR, Moayeri A: Mild hypothermia alters propofol pharmacokinetics and increases the duration of action of atracurium. Anesth Analg 80:10071014,1995

9. Nathan SS, Sinha SR, Gordon B, Lesser RP, Thakor NV: Determination of current density distributions generated by electrical stimulation of the human cerebral cortex. Electroencephalogr Clin Neurophysiol 86:183-192, 1993

10. Organ L, Tasker RR, Moody NF: Brain tumor localization using an electrical impedance technique. J Neurosurg 28:35-44, 1968

11. Prabhu SS, Gasco J, Tummala S, Weinberg JS, Rao G: Intraoperative magnetic resonance imaging-guided tractography with integrated monopolar subcortical functional mapping for resection of brain tumors. Clinical article. J Neurosurg 114:719-726, 2011

12. Shiban E, Krieg SM, Haller B, Buchmann N, Obermueller $\mathrm{T}$, Boeckh-Behrens T, et al: Intraoperative subcortical motor evoked potential stimulation: how close is the corticospinal tract? J Neurosurg 123:711-720, 2015

13. Smith JS, Cha S, Mayo MC, McDermott MW, Parsa AT, Chang SM, et al: Serial diffusion-weighted magnetic resonance imaging in cases of glioma: distinguishing tumor recurrence from postresection injury. J Neurosurg 103:428438,2005

14. Ueno A, Karashima A, Nakao M, Katayama N: Development of distance-selective nerve recruitment for subcortical brain mapping by controlling stimulation waveforms. Conf Proc IEEE Eng Med Biol Soc 2013:1879-1882, 2013
15. Ulmer S, Braga TA, Barker FG II, Lev MH, Gonzalez RG, Henson JW: Clinical and radiographic features of peritumoral infarction following resection of glioblastoma. Neurology 67:1668-1670, 2006

16. Yamaguchi F, Oi Y, Aoki W, Nakamura R, Igarashi A, Kubota M, et al: [BIS monitoring is useful for reliable intraoperative cortical mapping during brain tumor operations.] No Shinkei Geka 30:1181-1188, 2002 (Jpn)

17. Yamaguchi F, Takahashi H, Teramoto A: Intra-operative detection of motor pathways using a simple electrode provides safe brain tumor surgery. J Clin Neurosci 14:1106-1110, 2007

18. Yamaguchi F, Takahashi H, Teramoto A: Navigation-assisted subcortical mapping: intraoperative motor tract detection by bipolar needle electrode in combination with neuronavigation system. J Neurooncol 93:121-125, 2009

19. Yingling CD, Ojemann S, Dodson B, Harrington MJ, Berger MS: Identification of motor pathways during tumor surgery facilitated by multichannel electromyographic recording. J Neurosurg 91:922-927, 1999

20. Zhang X, Liu J, He B: Magnetic-resonance-based electrical properties tomography: a review. IEEE Rev Biomed Eng 7:87-96, 2014

\section{Disclosures}

The authors report no conflict of interest concerning the materials or methods used in this study or the findings specified in this paper.

\section{Author Contributions}

Conception and design: Yamaguchi. Acquisition of data: Yamaguchi, Ten, Higuchi, Omura, Kojima. Analysis and interpretation of data: Yamaguchi. Drafting the article: Yamaguchi. Critically revising the article: Yamaguchi. Reviewed submitted version of manuscript: Yamaguchi. Approved the final version of the manuscript on behalf of all authors: Yamaguchi. Administrative/techni$\mathrm{cal} /$ material support: Adachi, Kitamura, Kobayashi, Takahashi, Teramoto, Morita.

\section{Supplemental Information \\ Previous Presentations}

Preliminary results of this work have previously been presented as part of an e-poster presentation at the CNS 2015 Annual Meeting in New Orleans, Louisiana.

\section{Correspondence}

Fumio Yamaguchi, Department of Neurosurgery for Community Health, Nippon Medical School, 1-1-5, Sendagi, Bunkyo-ku, Tokyo 113-8603, Japan. email: fyamaguc@nms.ac.jp. 